\title{
TITLE:
}

\section{Projection and ground state correlations made simple}

AUTHOR(S):

Hagino, K; Reinhard, PG; Bertsch, GF

CITATION:

Hagino, K ... [et al]. Projection and ground state correlations made simple. PHYSICAL REVIEW C 2002, 65(6): 064320.

ISSUE DATE:

2002-06

URL:

http://hdl.handle.net/2433/50490

RIGHT:

Copyright 2002 American Physical Society 
PHYSICAL REVIEW C, VOLUME 65, 064320

\title{
Projection and ground state correlations made simple
}

\author{
K. Hagino, ${ }^{1}$ P.-G. Reinhard, ${ }^{2,3}$ and G. F. Bertsch ${ }^{2}$ \\ ${ }^{1}$ Yukawa Institute for Theoretical Physics, Kyoto University, Kyoto 606-8502, Japan \\ ${ }^{2}$ Institute for Nuclear Theory and Department of Physics, University of Washington, Seattle, Washington 98195 \\ ${ }^{3}$ Institut für Theoretische Physik II, Universität Erlangen-Nürnberg, Staudtstrasse 7, D-91058 Erlangen, Germany
}

(Received 26 February 2002; published 11 June 2002)

\begin{abstract}
We develop and test efficient approximations to estimate ground state correlations associated with low- and zero-energy modes. The scheme is an extension of the generator coordinate method (GCM) within Gaussian overlap approximation (GOA). We show that the GOA fails in non-Cartesian topologies and presents a topologically correct generalization of the GOA (topGOA). A random-phase-approximation like (RPA-like) correction is derived as the small amplitude limit of topGOA, called topRPA. Using exactly solvable models, the topGOA and topRPA schemes are compared with conventional approaches (GCM-GOA, RPA, Lipkin-Nogami projection) for rotational-vibrational motion and for particle-number projection. The results shows that the new schemes perform very well in all regimes of coupling.
\end{abstract}

DOI: 10.1103/PhysRevC.65.064320

PACS number(s): 21.60.Jz, 21.10.Dr

\section{INTRODUCTION}

Self-consistent mean-field models are nowadays the standard tool for nuclear structure calculations. Their quality has reached a level where one needs to take into account correlation effects beyond the mean field, particularly those which are related to low-energy or symmetry modes. Typical examples are center-of-mass projection, particle-number projection, angular momentum projection, or quadrupole surface vibrations. There are a large variety of techniques to deal with those correlations; for a review, see [1]. The most widely used ones are the random phase approximation (RPA) (see, e.g., [2,3]) and the generator coordinate method (GCM) (see, e.g., $[4,5])$. The latter has close links to projection formulas. The RPA has the advantage that it provides simple equations because it employs only second-order commutators of the basic one-body operators with the Hamiltonian. However, it runs into difficulties with soft modes which arise typically near transition points. The GCM is very general and extremely robust, but also very cumbersome to handle. Thus one has developed simplifications in the aim to use also preferably second-order expressions. This is achieved by the Gaussian overlap approximation (GOA) to the GCM; for details see the review in [4]. The GCM-GOA is a fair compromise between the generality of the GCM and the simplicity of the RPA. It uses up to second-order anticommutators but can still deal with large-amplitude collective motion. Second-order approximations within the spirit of the GOA have also been widely applied to projection schemes. The standard recipe $E_{\text {c.m. }}=\left\langle\hat{P}_{\text {c.m. }}^{2}\right\rangle / 2 m A$ for center-of-mass correction belongs to this class [6]. The similarly simple rotational correction $\left\langle\hat{J}^{2}\right\rangle / 2 \Theta$ has been widely employed, e.g., in the large-scale fits of [7]. And there is the well-known Lipkin-Nogami approach for particle-number projection [8].

However, one has to be aware that the GOA is not always performing well. For example, it fails for rotational motion in weakly deformed systems and for particle-number projection in the regime of weak pairing. The failure can be related to the topology of the collective coordinate under consider- ation. The GOA is well suited for Cartesian coordinates which extend in the interval $(-\infty,+\infty)$ with constantvolume element. The best example is here center-of-mass motion. But the GOA is not necessarily appropriate for other topologies such as, e.g., rotational motion whose coordinates are defined on a sphere. It can still work if the overlaps are falling off very quickly. But regimes of weak coupling have broad overlaps and thus the topology of the underlying coordinates is fully explored. It needs to be built into the approximation. An example for rotational motion is found in [9]. A most general construction for any topology is discussed in [10]. The changes are, in fact, obvious and simple. It amounts to building the topology of the coordinates into the parametrization of the GOA. We call the emerging approach a topologically corrected Gaussian overlap approximation (topGOA).

It is the aim of this paper to investigate the accuracy of the topGOA for two cases most relevant in nuclear structure calculations: deformations and particle-number projection. We compare the topGOA with the RPA as well as the full GCM and simple GOA. Furthermore, we derive a smallamplitude limit of the topGOA which gives at the end very simple and compact formulas for the collective ground state correlations, in a sense comparable to the RPA. We call that approach the topRPA. In both test cases we employ a suitable generalization of the Lipkin-Meshkov-Glick model [11].

\section{CONVENTIONAL APPROACHES}

This section provides a brief summary of traditionally well-known approaches for collective correlations, the RPA and GCM up to the GOA.

\section{A. RPA correlations}

The RPA theory is perhaps the most straightforward treatment of correlations beyond mean-field theory. It gives the leading corrections in the limit of a large number of interacting particles. With the RPA, one calculates an excitation spectrum of eigenfrequencies $\omega_{n}$ and the associated particle- 
hole operators $\hat{C}_{n}^{\dagger}$ that generate the eigenmodes. These modes are also present in the RPA ground state as zero-point motion, leading to a RPA theory of the ground state correlation energy; see, e.g., [2,12]. For a single mode, the RPA correlation energy is given by

$$
\Delta E=\frac{\omega}{2}\left(1-\left\langle\Phi_{0}\left|\left\{\hat{C}, \hat{C}^{\dagger}\right\}\right| \Phi_{0}\right\rangle\right),
$$

where

$$
\omega=\left\langle\Phi_{0}\left|\left[\hat{C},\left[\hat{H}, \hat{C}^{\dagger}\right]\right]\right| \Phi_{0}\right\rangle
$$

and $\left|\Phi_{0}\right\rangle$ is the mean-field ground state. In the case the mode corresponds to a broken continuous symmetry, $\omega=0$ and the formula should be applied by taking the $\omega \rightarrow 0$ limit. It is also advantageous in that case to separate the generators into time-even and time-odd generators

$$
\hat{Q}=\frac{1}{\sqrt{2}}\left(\hat{C}^{\dagger}+\hat{C}\right), \quad \hat{P}=\frac{i}{\sqrt{2}}\left(\hat{C}^{\dagger}-\hat{C}\right) .
$$

The $\hat{P}$ is usually the generator of a collective deformationfor example, a center-of-mass shift in case of the translational mode. Particle-number projection is an example where the time-even operator $\hat{N}$ spans the collective space.

The RPA correlation energy (1) can fail due to double counting if one employs a sum over a large RPA spectrum [13], but double counting is negligible if only a few collective modes are used [14]. That is the line of approach followed here. For a most recent survey of RPA correlations along that line, see [15]. It will be taken up explicitly in the applications later on.

\section{B. Generator coordinate method}

\section{General framework}

The most general technique for constructing collective modes is the generator coordinate method. It utilizes a superposition of wave functions defined along some collective deformation path $\left\{\left|\Phi_{q}\right\rangle \equiv|q\rangle\right\}$. Each state $|q\rangle$ along this path is an independent particle state (or independent quasiparticle state in case of BCS). The correlated wave function is given by

$$
|\Psi\rangle=\int d q|q\rangle f(q)
$$

where the superposition function $f$ is determined by the Griffin-Hill-Wheeler equation

$$
\begin{gathered}
\int d q^{\prime}\left[\mathcal{H}\left(q, q^{\prime}\right)-E \mathcal{I}\left(q, q^{\prime}\right)\right] f\left(q^{\prime}\right)=0, \\
\mathcal{H}\left(q, q^{\prime}\right)=\left\langle q|\hat{H}| q^{\prime}\right\rangle, \\
\mathcal{I}\left(q, q^{\prime}\right)=\left\langle q \mid q^{\prime}\right\rangle .
\end{gathered}
$$

Normalizing $\Psi$, the correlation energy is given by

$$
\Delta E=\langle\Psi|\hat{H}| \Psi\rangle-\left\langle\Phi_{0}|\hat{H}| \Phi_{0}\right\rangle,
$$

where $\left|\Phi_{0}\right\rangle \equiv|0\rangle$ is the ground state of the underlying independent particle model. The GCM can be easily generalized to multiple modes. One simply generalizes $q$ to a vector of deformations and extends $\int d q^{\prime}$ to a multidimensional integral; see, e.g., $[4,16,17]$. The GCM is often applied in this straightforward, but tedious, manner where the overlaps and the solution of the Griffin-Hill-Wheeler equation are determined numerically; see, e.g., $[5,18,19]$.

\section{Gaussian overlap approximation}

The full GCM is much more elaborate than the RPA because one deals with the overlaps for any combination of $q$ and $q^{\prime}$ and the highly nonlocal Griffin-Hill-Wheeler equation. A dramatic simplification is achieved by the Gaussian overlap approximation. It represents the dependence of the overlaps on the difference $\left(q-q^{\prime}\right)$ by a Gaussian times a polynomial in $\left(q-q^{\prime}\right)^{n}$. The overlap is represented as a pure Gaussian,

$$
\mathcal{I}\left(q, q^{\prime}\right)=\exp \left(-\frac{\lambda}{4}\left(q-q^{\prime}\right)^{2}\right),
$$

with

$$
\lambda(\bar{q})=\left.\frac{1}{2}\left(i \partial_{q}-i \partial_{q^{\prime}}\right)^{2} \mathcal{I}\left(q, q^{\prime}\right)\right|_{q=q^{\prime}=\bar{q}}
$$

One usually goes up to second-order derivatives in the expression for the Hamiltonian:

$$
\begin{gathered}
\frac{\mathcal{H}\left(q, q^{\prime}\right)}{\mathcal{I}\left(q, q^{\prime}\right)}=\mathcal{H}_{0}(\bar{q})-\frac{1}{8}\left(q-q^{\prime}\right)^{2} \mathcal{H}_{2}(\bar{q}), \\
\mathcal{H}_{0}(\bar{q})=\mathcal{H}(\bar{q}, \bar{q}), \\
\mathcal{H}_{2}(\bar{q})=\left.\left(i \partial_{q}-i \partial_{q^{\prime}}\right)^{2} \frac{\mathcal{H}\left(q, q^{\prime}\right)}{\mathcal{I}\left(q, q^{\prime}\right)}\right|_{q=q^{\prime}=\bar{q}}, \\
\bar{q}=\frac{q+q^{\prime}}{2} .
\end{gathered}
$$

For further details, see [4]. The GOA yields a dramatic simplification of the Griffin-Hill-Wheeler equation. Assuming that the coefficients depend only weakly on $\bar{q}$, one can recast the Griffin-Hill-Wheeler equation into a collective Schrödinger equation with a simple second derivative as operator for the kinetic energy. Large amplitudes in average collective deformation $\bar{q}=\left(q+q^{\prime}\right) / 2$ are still allowed. Thus the GCMGOA is applicable to conditions of large fluctuation as are typical for low-energy modes and for symmetry projection.

A further dramatic simplification emerges if one restricts the considerations to small amplitudes also in $\bar{q}=(q$ $\left.+q^{\prime}\right) / 2$. Then the collective dynamics becomes harmonic and all expressions can be worked out analytically. The final result is then just the RPA $[16,14,17]$. The correlations from 
the GCM-GOA become then identical to the RPA correlations as given the above section II A.

\section{Beyond the GOA}

However, the GOA has its limitations. The Gaussian ansatz assumes tacitly that the collective coordinate spans the interval

$$
q \in(-\infty,+\infty)
$$

In other words, the dynamics is fundamentally Cartesian in the collective coordinates. This is certainly true for some situations, e.g., the center-of-mass motion where each coordinate $R_{x}, R_{y}$, and $R_{z}$ runs over $(-\infty,+\infty)$. But the presupposition is violated in many cases. In particular, in rotational motion the rotation angles are restricted to finite intervals with periodic boundary conditions. For such situations the GOA can be generalized by modifying the arguments of the Gaussian to correctly include the topology of the collective mode $[9,10]$. We call this generalization the topological GOA (topGOA). The details of topGOA depend, of course, on the actual mode under considerations. In the following, we exemplify and test the topGOA for two typical and most important applications in nuclear physics: deformations and particle-number projection. The projection is straightforward and yields immediately expressions in second order throughout. The efficient treatment of deformations remains an important problem in nuclear structure. The theory should provide accurate correlation energies, going from the smallamplitude vibrational limit to the large-amplitude static deformations and including the soft region in between. These applications will serve as a critical testing ground for the topRPA and the small-amplitude approximation to the topGOA.

\section{VIBRATIONS AND ROTATIONAL PROJECTION}

\section{A. Three-level model}

The usual two-level Lipkin-Meshkov-Glick Hamiltonian has been widely used to model the collective motion of in a deformation coordinate, as it contains the vibrational and static deformation limits with the mean-field phase transition in between. However, the model does not have a continuous symmetry, which is an important aspect of the deformations. To include a continuous symmetry, we have extended the space in the Lipkin-Meskov-Glick model to three levels and call the extended model the three-level model. Two of the levels are degenerate in the three-level model, and the interactions treat those levels identically. This introduces a symmetry mode with the topology of rotations in a plane. For clarity we repeat here the definition of the three-level model; for details, see [15]. The three levels are labeled 0,1 , and 2. The basic $1 p h$ transitions $0 \rightarrow 1$ are induced by $\hat{K}_{+, 1}$ and those to state 2 by $\hat{K}_{+, 2}$. The amount of excitation is measured by $\hat{K}_{0, i}, i \in\{1,2\}$. The $\hat{K}$ operators obey a quasispin algebra. The Hamiltonian of the model reads

$$
\begin{gathered}
\hat{H}=\epsilon \sum_{i=1}^{2} \hat{K}_{0, i}-\chi \frac{\epsilon}{2(N-1)} \sum_{i}\left(\hat{K}_{+, i}^{2}+\hat{K}_{-, i}^{2}\right), \\
\hat{K}_{0, i}=\sum_{m=1}^{N} a_{i m}^{\dagger} a_{i m}, \\
\hat{K}_{+, i}=\sum_{m=1}^{N} a_{i m}^{\dagger} a_{0 m}, \\
\hat{K}_{-, i}=\sum_{m=1}^{N} a_{0 m}^{\dagger} a_{i m},
\end{gathered}
$$

The exact solution of this Hamiltonian is obtained by diagonalization in the space of $\hat{K}_{x}^{n} \hat{K}_{y}^{m}$. The three-level Hamiltonian is the first term in Eq. (11a). It defines the energetic relations among the levels. Note that the two excited states $i=1,2$ are degenerate. This gives the model the rotational symmetry. The second term in Eq. (11a) models a two-body interaction. It is again symmetric in $i=1 \leftrightarrow 2$ which maintains rotational symmetry. The strength is regulated by $\chi$, defined to be the dimensionless coupling strength. We will see later that $\chi \sim 1$ is the critical point in the model separating weak and strong coupling.

It is convenient to analyze the many-particle wave function in terms of collective variables $\alpha$ and $\beta$. The collective wave function is defined as

$$
|\alpha \beta\rangle=e^{\tan (\alpha) \hat{K}_{+}(\beta)}|0\rangle \mathcal{N}^{-1 / 2}(\alpha),
$$

where

$$
\hat{K}_{+}(\beta)=\cos (\beta) \hat{K}_{+, 1}+\sin (\beta) \hat{K}_{+, 2}
$$

and the normalization is given by

$$
\mathcal{N}(\alpha)=\left\langle 0\left|e^{\tan (\alpha) \hat{K}_{-}} e^{\tan (\alpha) \hat{K}_{+}}\right| 0\right\rangle=\cos ^{-2 N}(\alpha) .
$$

Note that the model is rotationally invariant in the angle $\beta$. The motion in $\alpha$ corresponds to collective vibrations. The system is close to a good vibrator for small residual interaction, $\chi<1$. It is a rigid rotator for large $\chi>1$. The transitional regime $\chi \sim 1$ explores collective motion with largeamplitude fluctuations. Two subtle details need to be mentioned: First, there is only one rotational degree of freedom which means that the model corresponds to rotations in a plane. Second, the vibrational degree of freedom contains relevant information only in the interval $\alpha \in\{0, \pi\}$, similar to the vibrational mode in the usual Lipkin-Meshkov-Glick model. This is the price one pays to have a simple model.

The simplicity of the model allows one to write down the exact overlaps analytically:

$$
\begin{aligned}
& \mathcal{I}\left(\alpha \beta, \alpha^{\prime} \beta^{\prime}\right) \\
& \quad=\left[\cos (\alpha) \cos \left(\alpha^{\prime}\right)+\sin (\alpha) \sin \left(\alpha^{\prime}\right) \cos \left(\beta-\beta^{\prime}\right)\right]^{N},
\end{aligned}
$$




$$
\begin{aligned}
& \frac{\mathcal{H}\left(\alpha \beta, \alpha^{\prime} \beta^{\prime}\right)}{\mathcal{I}\left(\alpha \beta, \alpha^{\prime} \beta^{\prime}\right)} \\
& =N \epsilon \frac{\sin \alpha \sin \alpha^{\prime} \cos \left(\beta-\beta^{\prime}\right)}{\cos \alpha \cos \alpha^{\prime}+\sin \alpha \sin \alpha^{\prime} \cos \left(\beta-\beta^{\prime}\right)} \\
& \quad-\chi \frac{\epsilon}{2} N \frac{\sin ^{2} \alpha \cos ^{2} \alpha^{\prime}+\cos ^{2} \alpha \sin ^{2} \alpha^{\prime}}{\left[\cos \alpha \cos \alpha^{\prime}+\sin \alpha \sin \alpha^{\prime} \cos \left(\beta-\beta^{\prime}\right)\right]^{2}} .
\end{aligned}
$$

The Hartree-Fock (HF) solution is obtained simply by minimizing the expectation value of the Hamiltonian in a state $|\alpha \beta\rangle$,

$$
\begin{aligned}
E_{\mathrm{mf}}(\alpha) & =\mathcal{H}(\alpha \beta, \alpha \beta) \\
& =N \epsilon \sin ^{2}(\alpha)-\chi \epsilon N \sin ^{2}(\alpha) \cos ^{2}(\alpha),
\end{aligned}
$$

with respect to the deformation $\alpha, \beta$. This yields the HartreeFock energy as $E_{\mathrm{HF}}=E_{\mathrm{mf}}\left(\alpha_{\mathrm{HF}}\right)$ where the deformation of the minimum is denoted by $\alpha_{\mathrm{HF}}$. Note that the energy is independent of the actual value of $\beta$ due to rotational invariance of the three-level model.

\section{B. RPA modes}

Small-amplitude motion around the HF minimum induces collective excitations of the system. They can be worked out analytically for the three-level model [15]. There are two collective modes to be considered. At spherical shape $\alpha_{\mathrm{HF}}$ $\sim 0$, there are two degenerate vibrational modes. The degeneracy is lifted with increasing $\alpha_{\mathrm{HF}}$. With further increasing $\alpha_{\mathrm{HF}}$, there comes a critical point where the RPA solutions become unstable. A different scenario develops after the transition point. The two modes separate into a rotational mode along $\beta$ and a vibrational mode along $\alpha$. The two eigenfrequencies are $\omega=0$, associated with the rotational mode, and $\omega=\epsilon \sqrt{\chi^{2}-1}$ for vibrations. Having these two modes at hand, one can compute the RPA correlation energy applying Eq. (1) for each mode separately and add up the result to the total correlations.

\section{TopGOA for the three-level model}

The standard GOA overlaps can be obtained by expanding Eq. (14) with respect to $\left(\alpha-\alpha^{\prime}\right)$ and $\left(\beta-\beta^{\prime}\right)$ up to second order. We exemplify it here for the norm kernel at $\alpha=\alpha^{\prime}$ and expansion in $\beta-\beta^{\prime}$. The GOA reads

$$
\begin{aligned}
\mathcal{I}\left(\alpha \beta, \alpha \beta^{\prime}\right) & =\left[\cos ^{2}(\alpha)+\sin ^{2}(\alpha) \cos \left(\beta-\beta^{\prime}\right)\right]^{N}, \\
& =\left[1-2 \sin ^{2}(\alpha) \sin ^{2}\left(\frac{\beta-\beta^{\prime}}{2}\right)\right]^{N} \\
& \rightarrow \exp \left(-\frac{N}{2} \sin ^{2}(\alpha)\left(\beta-\beta^{\prime}\right)^{2}\right)
\end{aligned}
$$

The problem is obvious: the exact overlap is periodic in $\beta$ $-\beta^{\prime}$ while the GOA is not.
To develop an appropriate ansatz for the topGOA we have to look at the topology of the collective coordinates. The pair of coordinates $(\alpha, \beta)$ extends over the surface of the unit sphere. The exact overlaps (14) hint already at the combination of coordinates which is generated by this topology: $\cos (\alpha) \cos \left(\alpha^{\prime}\right)+\sin (\alpha) \sin \left(\alpha^{\prime}\right) \cos \left(\beta-\beta^{\prime}\right)$. It is the measure for a distance on the sphere. The idea of the topGOA is to apply to the norm overlap the Gaussian limit theorem for the shape of the overlap function while preserving the topological combination of the arguments. Similar combinations are to be used for expanding the Hamiltonian overlap. This yields then for the three-level model the form

$$
\begin{aligned}
& \mathcal{I}\left(\alpha \beta, \alpha^{\prime} \beta^{\prime}\right)=\exp \left[-\frac{\lambda_{\alpha}}{4} \sin ^{2}\left(\frac{\alpha-\alpha^{\prime}}{2}\right)\right. \\
& \left.-\frac{\lambda_{\beta}}{4} \mathcal{S}_{\alpha} \sin ^{2}\left(\frac{\beta-\beta^{\prime}}{2}\right)\right] \text {, } \\
& \lambda_{\alpha}=\left.\frac{1}{2}\left(i \partial_{\alpha}-i \partial_{\alpha^{\prime}}\right)^{2} \mathcal{I}\left(\alpha \beta, \alpha^{\prime} \beta\right)\right|_{\alpha=\alpha^{\prime}=\bar{\alpha}} \\
& \lambda_{\beta} \mathcal{S}_{\alpha}=\left.\frac{1}{2}\left(i \partial_{\beta}-i \partial_{\beta^{\prime}}\right)^{2} \mathcal{I}\left(\alpha \beta, \alpha \beta^{\prime}\right)\right|_{\beta=\beta^{\prime}=\bar{\beta}} \\
& \frac{\mathcal{H}\left(\alpha \beta, \alpha^{\prime} \beta^{\prime}\right)}{\mathcal{I}\left(\alpha \beta, \alpha^{\prime} \beta^{\prime}\right)}=\mathcal{H}_{0}-\mathcal{H}_{2}^{\alpha} \sin ^{2}\left(\frac{\alpha-\alpha^{\prime}}{2}\right) \\
& -\mathcal{H}_{2}^{\beta} \mathcal{S}_{\alpha} \sin ^{2}\left(\frac{\beta-\beta^{\prime}}{2}\right), \\
& \mathcal{H}_{0}=\mathcal{H}(\bar{\alpha} \bar{\beta}, \bar{\alpha} \bar{\beta}) \\
& \mathcal{H}_{2}^{\alpha}=\left.\frac{-1}{2}\left(\partial_{\alpha}-\partial_{\alpha^{\prime}}\right)^{2} \frac{\mathcal{H}\left(\alpha \beta, \alpha^{\prime} \beta\right)}{\mathcal{I}\left(\alpha \beta, \alpha^{\prime} \beta\right)}\right|_{\alpha=\alpha^{\prime}=\bar{\alpha}} \\
& \mathcal{H}_{2}^{\beta}=\left.\frac{-1}{2 \mathcal{S}_{\alpha}}\left(\partial_{\beta}-\partial_{\beta^{\prime}}\right)^{2} \frac{\mathcal{H}\left(\alpha \beta, \alpha \beta^{\prime}\right)}{\mathcal{I}\left(\alpha \beta, \alpha \beta^{\prime}\right)}\right|_{\beta=\beta^{\prime}=\bar{\beta}} \\
& \mathcal{S}_{\alpha}=\bar{s}^{2}-\sin ^{2}\left(\frac{\alpha-\alpha^{\prime}}{2}\right) \\
& \bar{s}=\sin (\bar{\alpha}), \quad \bar{c}=\cos (\bar{\alpha}), \\
& \bar{\alpha}=\frac{\alpha+\alpha^{\prime}}{2} .
\end{aligned}
$$

Thus far we have the topGOA overlaps for any system where the collective coordinates form the topology of a sphere. The specific coefficients for the present three-level model are

$$
\begin{gathered}
\lambda_{\alpha}=8 N, \\
\lambda_{\beta}=8 N, \\
\mathcal{H}_{0}=N \epsilon \bar{S}^{2}-N \chi \epsilon \bar{S}^{2} \bar{c}^{2},
\end{gathered}
$$




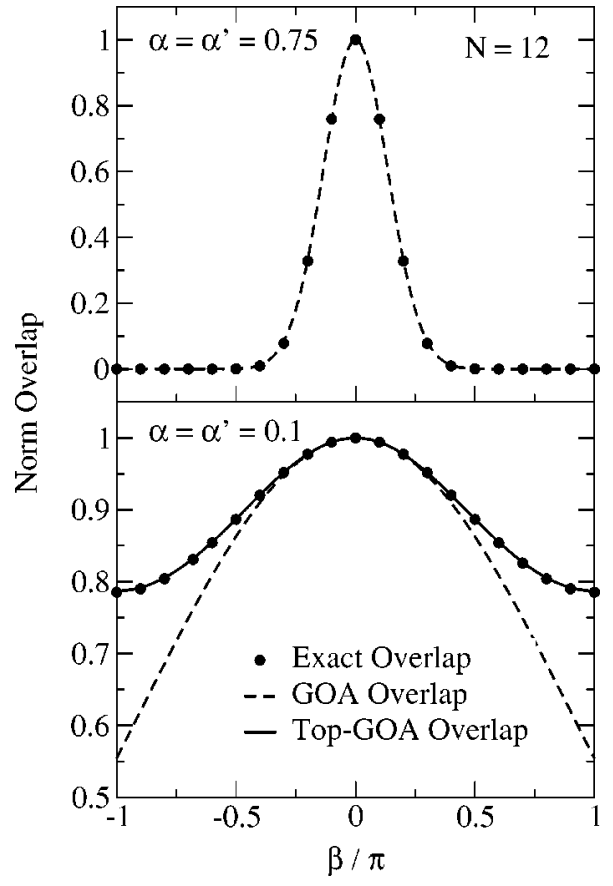

FIG. 1. The norm overlaps along the $\beta$ direction for two different deformations $\alpha$ as indicated, for the three-level model with $N$ $=12$. The dots show the exact overlaps, dashed lines stand for the standard GOA, and solid line for the topGOA. The topGOA is not shown in the upper panel because it is graphically identical with the standard GOA.

$$
\begin{gathered}
\mathcal{H}_{2}^{\alpha}=N \epsilon\left(\bar{c}^{2}-\bar{s}^{2}\right)+N \chi \epsilon\left(4 \bar{s}^{2} \bar{c}^{2}+1\right), \\
\mathcal{H}_{2}^{\beta}=2 N \epsilon \bar{c}^{2}+4 N \chi \epsilon \bar{s}^{2} \bar{c}^{2} .
\end{gathered}
$$

The effect of the GOA versus topGOA for the norm overlap is demonstrated in Fig. 1. For large deformations (upper panel), the norm overlap decays rather quickly in angle $\beta$. The conventional GOA is here a reliable approximation. The situation is much different at small deformation. The overlaps become broad and hit the periodicity limits. This yields a dramatic difference between the GOA and topGOA. Note that the topGOA is still an excellent approximation to the exact overlap while the GOA fails badly.

\section{Performance of the topGOA}

The conventional GOA, Eq. (9a), maps the Griffin-HillWheeler equation (5) onto a collective Schrödinger equation of second order in the collective momentum [4,5]. This feature is lost in the topGOA. Further approximation steps would be needed to come to that end. We will not pursue them further here and solve directly the Griffin-Hill-Wheeler equation (5) inserting the topGOA overlaps (16).

Figure 2 compares the RPA and topGOA with the HF and exact results for a large variety of coupling strengths. The uppermost panel shows total energies. One sees that both approaches correct the HF energy very far towards the exact energy. However, the RPA shows irregularities near the critical point $\chi \approx 1$.

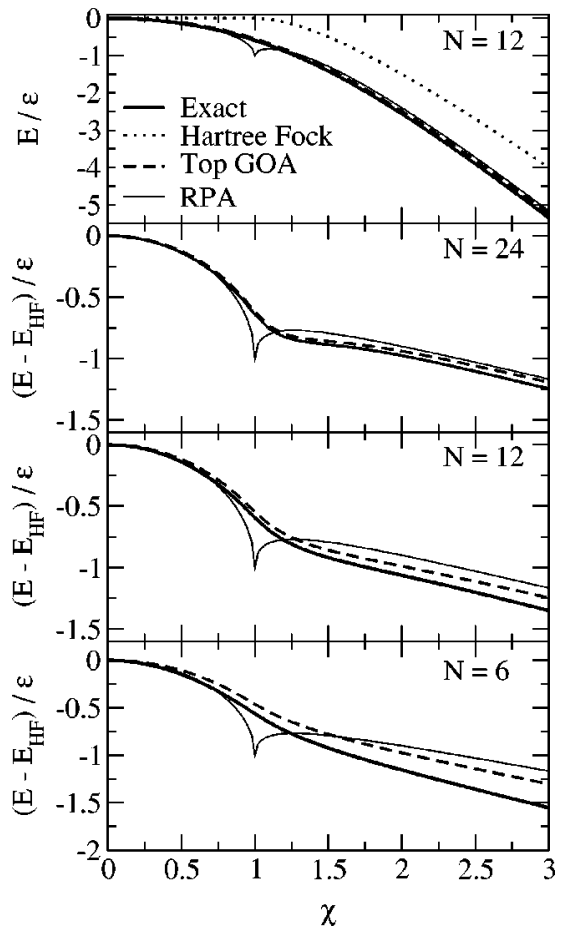

FIG. 2. Comparison of energies for the three-level model at various levels of approximations as indicated. Upper block: total energies for $N=12$. Lower block: the correlation energy $\Delta E=E$ $-E_{\mathrm{HF}}$ for various $N$ as indicated. Results are drawn for a large range of coupling strengths $\chi$ from sphericity $\chi=0$ deeply into the deformed regime.

A more detailed look is given in the three lower panels of Fig. 2 where we show the correlation energies $\Delta E=E$ $-E_{\mathrm{HF}}$ for the various approaches and for a series of system sizes. The RPA provides a useful correction in the limits of sphericity and well-developed deformations, but fails badly around the critical point. The topGOA performs very well in all regimes. The results improve with increasing system size as one could expect from the Gaussian limit theorem inherent in the topGOA. Acceptable results are obtained from the topGOA also for $N=4$. But all approaches become inaccurate for $N=2$ which is obviously not collective enough.

\section{E. Angular momentum projection}

When the mean-field ground state breaks a symmetry of the Hamiltonian, one can get an improved wave function and energy by projection, i.e., take a minimal set of states $q$ and appropriate $f$ in Eq. (4) to enforce the symmetry. This is particularly useful for deformations and projection of the $J$ $=0$ ground state out of a deformed intrinsic state. The questions before us are, how does this technique compare with the RPA or the topGOA for computing the correlation energy? It should be noted that the projection method has a formal advantage in that the calculated energy is an upper bound of the true energy associated with the Hamiltonian.

\section{Projected state}

We will examine how well the projection technique works for the three-level model as a test case. Rotational projection on the ground state angular momentum $M=0$ reads simply 


$$
|\alpha\rangle_{\mathrm{proj}} \propto \int_{-\pi}^{\pi} d \beta|\alpha \beta\rangle .
$$

The rotationally projected energy is computed as the expectation value which amounts to integrating the overlaps over the angular coordinate $\beta$, i.e.,

$$
E_{\mathrm{proj}}(\alpha)=\frac{\int d\left(\beta-\beta^{\prime}\right) \mathcal{H}\left(\alpha \beta, \alpha \beta^{\prime}\right)}{\int d\left(\beta-\beta^{\prime}\right) \mathcal{I}\left(\alpha \beta, \alpha \beta^{\prime}\right)} .
$$

This is simple and straightforward for the topGOA overlaps of the form (16). We thus can skip the details.

\section{Variation before and after projection}

The energy (19) can be computed for any given deformation $\alpha$. The HF ground state deformation $\alpha_{\mathrm{HF}}$ is obtained from minimizing the mere HF energy (16e). Applying the projection on this state corresponds to the scheme "variation before projection." It serves to correct for the angular momentum fluctuations in the deformed HF ground state. A much better approach is obtained when performing "variation after projection" [12]. Here one minimizes the projected energy (19). This is an involved task for exact projection. The topGOA approach yields a simple expression for the projected energy on which a variation is still feasible. It is, of course, particularly simple in the present test case. We just have to search for the deformation $\alpha_{\text {proj }}$ which minimizes $E_{\text {proj }}$.

The variation-before-projection and variation-afterprojection schemes are compared in Fig. 3 for a large range of coupling strengths. The upper panel shows the ground state deformations. The variation-before-projection state stays spherical up the critical point and switches to a deformation with a discontinuous derivate (second-order transition). The variation-after-projection states develop more smoothly and show a steady growth of deformation. The variation-after-projection scheme can afford intrinsic deformations because it "knows" that projection will restore spherical symmetry. The freedom which the variation-afterprojection scheme exploits will yield a lower energy. This is shown in the lower panel of Fig. 3. It is obvious that the variation-after-projection scheme picks up a large fraction of the correlation energy at any coupling strength $\chi, 80 \%$ for strongly deformed systems and even more for weakly deformed ones. This makes it obvious that the variation-afterprojection scheme is the superior strategy. Note that the topGOA helps to simplify the variation-after-projection scheme considerably. We will test it now in the next paragraph.

\section{Performance of the topGOA for a.m. projection}

The performance of the topGOA for rotational projection is checked in Fig. 4 for the case of $N=12$. The conventional GOA has obviously problems at small deformation up to beyond the critical point. But the topGOA provides a very good approximation to exact projection throughout. And it does that on the grounds of a simple expression for the pro-

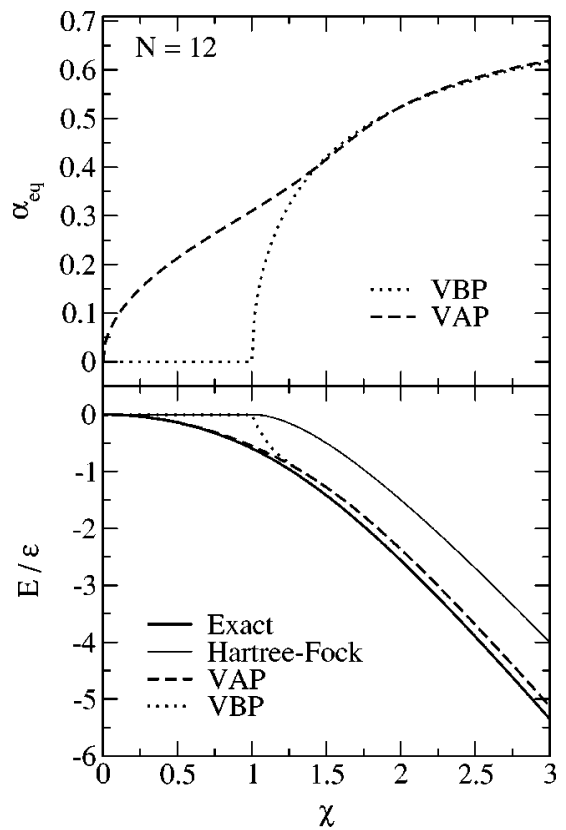

FIG. 3. Comparison of variation after projection (VAP) and variation before projection (VBP) in the three-level model with $N$ $=12$ using exact projection. Upper panel: ground state deformation $\alpha_{\text {eq }}$. Lower panel: ground state energies from HF and from rotational projection both ways, compared with the exact energy.

jected energy which can be deduced from second-order moments only. This is welcome for an efficient variation after projection and it is particularly helpful in connection with effective energy functionals because double (anti)commuta-

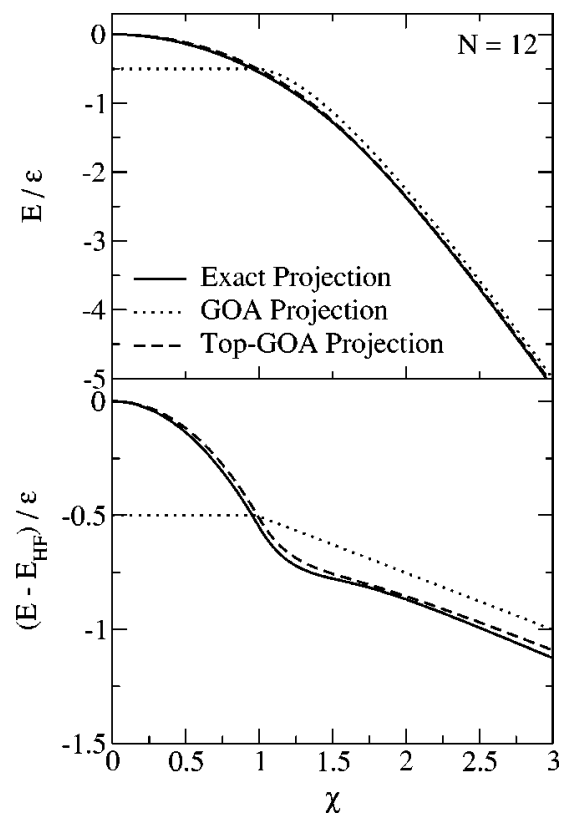

FIG. 4. The correlation energy $\Delta E=E-E_{\mathrm{HF}}$ at various levels of approximation for the three-level model $N=12$. The compared cases are full $=$ exact projection energy, dotted = projection using standard GOA, dashed = projected energy using topGOA. The upper panel shows the energies as such and the lower panel shows the correlation energy, i.e., the difference to the mere HF energy. 
tors with $\hat{H}$ can still be safely derived from second functional derivatives; see Sec. IV E.

The rotational projection can still be done with secondorder information around the minimum point. It is thus as simple to compute as the RPA. And this simple part provides the dominant portion of the correlation energy. The most costly part of the correlation energy is computing the small final contribution from vibrations. It is tempting to consider mere angular projection as a first guess for the correlation energy. That is, in fact, a strategy pursued in the large-scale fits of [7]. Our result here provides a welcome substantiation of their "rule of thumb."

\section{F. thoroughly second-order approach: The topRPA}

The conclusions from the previous subsection encourage a quest for a more efficient estimator of the vibrational correlation energy. And the typical pattern of the variation-afterprojection scheme adds reasons to that. We have seen in Fig. 3 that the variation-after-projection ground state is nearly always deformed. The projected energy as function of $\alpha$ has always a fairly well-developed minimum much in contrast to the HF energy which is rather soft around the critical point. This hints that one is allowed to perform a small-amplitude expansion about the projected minimum $\alpha_{\text {proj }}$. Once having accepted this idea, the remaining steps are obvious and simple.

(1) One performs variation after projection using the topGOA for rotational projection. This yields the variation-afterprojection ground state deformation $\alpha_{0}$.

(2) One computes the topGOA projected energy

$$
E_{\text {proj }}(\bar{\alpha})=E_{\text {proj }}\left(\alpha_{0}\right)+\frac{1}{2}\left(\bar{\alpha}-\alpha_{0}\right)^{2} \partial_{\bar{\alpha}}^{2} E_{\text {proj }}\left(\alpha_{0}\right)
$$

in the vicinity of $\alpha_{0}$ and deduces the curvature $\partial_{\alpha}^{2} E_{\text {proj }}$ of this effective potential.

(3) For the remaining vibrational correction, one applies the simple correlation energy from the harmonic approximation

$$
\begin{gathered}
\delta E_{\text {vib }}=\frac{1}{2} \sqrt{\partial_{\alpha}^{2} E_{\text {proj }} \mathcal{B}}-\left(\frac{\partial_{\alpha}^{2} E_{\text {proj }}}{4 \lambda_{\text {proj }}}+\frac{\lambda_{\text {proj }} \mathcal{B}}{4}\right), \\
\mathcal{B}=\frac{2 \mathcal{H}_{2}^{\alpha}\left(\alpha_{0}\right)}{\lambda_{\text {proj }}^{2}}, \\
\lambda_{\text {proj }}=\left.2 \partial_{\alpha} \partial_{\alpha^{\prime}}\left\langle\alpha^{\prime} \mid \alpha\right\rangle_{\text {proj }}\right|_{\alpha=\alpha^{\prime}=\alpha_{0},} \\
\mathcal{H}_{2}^{\alpha}\left(\alpha_{0}\right)=\left.2 \partial_{\alpha} \partial_{\alpha^{\prime}} \frac{\left\langle\alpha^{\prime}|\hat{H}| \alpha\right\rangle_{\text {proj }}}{\left\langle\alpha^{\prime} \mid \alpha\right\rangle_{\text {proj }}}\right|_{\alpha=\alpha^{\prime}=\alpha_{0}} .
\end{gathered}
$$

(4) The total energy is then finally

$$
E=\delta E_{\text {proj }}\left(\alpha_{0}\right)-\delta E_{\text {vib }} .
$$

Note that this scheme requires only information on secondorder derivatives in $\alpha$ and $\beta$ about the deformed ground

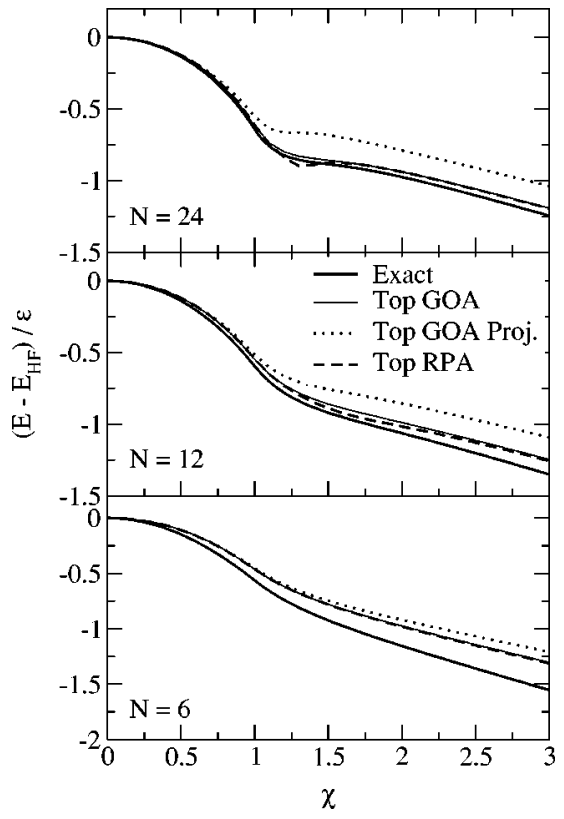

FIG. 5. Comparison of the topGOA and topRPA for the threelevel model and various $N$ as indicated. Upper block: total energies compared with HF and the exact result. Lower block: the correlation energies $E_{\text {scheme }}-E_{\mathrm{HF}}$ for various levels of approximation. Here "scheme" stands for the exact solution (solid line), the topGOA (dotted line), the topRPA (dashed line). Results are drawn vs effective coupling strength $\chi$.

state. In that sense it is much similar to the RPA. We thus call that scheme the topologically corrected RPA (topRPA). The essence is, of course, that topological constraints are exploited to construct from the given second-order information the final ground state energy in the topRPA.

Figure 5 compares the performance of the topRPA and topGOA for the correlation energy in the three-level model. It is obvious that the topRPA provides a good approximation to the topGOA, equally good for all system sizes. Both schemes constitute a reliable approach to the exact result, better for larger systems. For completeness, we show also the correlation from angular momentum projection alone. We see again that this exhausts the leading part of correlations and could be considered as a quick and simple approach. However, the topRPA is not much more expensive and comes close to the final result.

\section{PARTICLE-NUMBER-PROJECTION}

The second test case in this paper is concerned with particle-number projection. It becomes necessary when starting Hartree-Fock-Bogoliubov (HFB) states, or its BCS approximation, are involved. The HFB approximation produces independent quasiparticle states which have mixed particle number $N$. One needs to project the HFB states onto a good particle number. This is important in any nuclear structure calculation because doubly magic nuclei (where mere HF suffices) are an extremely rare species. Similar as in the previous example of rotation-vibrations there is, in principle, a pair of modes: namely, particle number projection and pair- 
ing vibrations. We confine here the discussion to projection alone because that is the widely used strategy and because it will again exhaust the dominant part of the correlations.

\section{A. Exact projection}

Let $\left|\Phi_{0}\right\rangle$ be a HFB state with average particle number:

$$
\left\langle\Phi_{0}|\hat{N}| \Phi_{0}\right\rangle=N_{0}
$$

The projected state with exact particle number $N_{0}$ is

$$
|\Psi\rangle \propto \int_{0}^{2 \pi} d \eta|\eta\rangle, \quad|\eta\rangle=e^{i \eta \tilde{N}}\left|\Phi_{0}\right\rangle
$$

where

$$
\widetilde{N}=\hat{N}-N_{0} .
$$

The construction of the path from straightforward $\exp (i \eta \tilde{N})$ makes the norm overlap a function of the difference alone, i.e., $\mathcal{I}=\mathcal{I}\left(\eta-\eta^{\prime}\right)$. The number conservation $[\hat{H}, \tilde{N}]=0$ causes also $\mathcal{H}=\mathcal{H}\left(\eta-\eta^{\prime}\right)$. The projected energy thus becomes

$$
E=\frac{\int d \eta\left\langle\Phi_{0}\left|\hat{H} e^{i \eta \tilde{N}}\right| \Phi_{0}\right\rangle}{\int d \eta\left\langle\Phi_{0}\left|e^{i \eta \tilde{N}}\right| \Phi_{0}\right\rangle}=\frac{\int d \eta \mathcal{H}(\eta)}{\int d \eta \mathcal{I}(\eta)} .
$$

\section{B. TopGOA for particle-number projection: Overlaps and correlation energy}

The collective path is $|\eta\rangle$ as given in Eq. (23a). The collective coordinate is defined in the interval $[0,2 \pi)$ and is periodic as $\eta \rightarrow \eta+2 \pi$. This periodicity is not reproduced by the standard GOA overlaps (9a). One has to modify the GOA to account for that structure; in other words, one has to employ the topologically correct GOA (topGOA). Taking up the experience from the previous test case, we can postulate that the periodic structure of the coordinates is properly taken into account by the argument in the GOA through

$$
\frac{\eta}{2} \rightarrow \sin \left(\frac{\eta}{2}\right)
$$

One may wonder why we use this particular assignment for the generalization. The choice is unique in that it corresponds to the base period of the squared sine function. Other fractions would not have the correct periodicity of the Hamiltonian. The generalized overlaps for particle-number projection are then

$$
\begin{gathered}
\mathcal{I}(\eta)=\exp \left(-2\left\langle\tilde{N}^{2}\right\rangle \sin ^{2}\left(\frac{\eta}{2}\right)\right), \\
\mathcal{H}(\eta)=\mathcal{I}(\eta)\left[\mathcal{H}_{0}-\frac{1}{2} \sin ^{2}\left(\frac{\eta}{2}\right) \mathcal{H}_{2}\right],
\end{gathered}
$$

$$
\begin{aligned}
& \mathcal{H}_{0}=\left\langle\Phi_{0}|\hat{H}| \Phi_{0}\right\rangle=E_{\mathrm{BCS}}, \\
& \mathcal{H}_{2}=\langle\{\tilde{N},\{\hat{H}-\langle\hat{H}\rangle, \tilde{N}\}\}\rangle .
\end{aligned}
$$

Note that the width $\lambda=2\left\langle\widetilde{N}^{2}\right\rangle$ and the coefficients $\mathcal{H}_{i}$ of the Hamiltonian overlap are still defined as in the standard GOA; see Eq. (9a). What changes is the way these overlaps are extrapolated. It is obvious that the conventional GOA is recovered in the case of a steeply decaying norm overlap, i.e., for $\lambda \rightarrow \infty$.

The projected energy (24) can then be expressed in rather compact fashion as

$$
E=E_{\mathrm{BCS}}-\delta E_{\mathrm{PNP}},
$$

$$
\delta E_{\mathrm{PNP}}=\frac{1}{4} \Lambda\left(\left\langle\widetilde{N}^{2}\right\rangle\right) \mathcal{H}_{2}
$$

$$
\Lambda(y)=\frac{\int_{0}^{2 \pi} d \eta e^{-2 y \sin ^{2}(\eta / 2)} 2 \sin ^{2}\left(\frac{\eta}{2}\right)}{\int_{0}^{2 \pi} d \eta e^{-2 y \sin ^{2}(\eta / 2)}} .
$$

In the limiting case, the standard GOA is recovered by

$$
\Lambda \rightarrow 1 /\left(2\left\langle\tilde{N}^{2}\right\rangle\right) \text { for }\left\langle\tilde{N}^{2}\right\rangle \rightarrow \infty .
$$

This corresponds to a HFB state deep in the pairing regime where one gathers substantial particle-number fluctuations. The opposite limit is

$$
\Lambda \rightarrow 1 \text { for }\left\langle\tilde{N}^{2}\right\rangle \rightarrow 0
$$

It corresponds to the breakdown of pairing towards a pure HF state. The standard GOA fails here. It is obvious that only the topGOA can cope properly with that pairing transition.

As in the case of angular momentum projection, there is the choice between the variation-before-projection and variation-after-projection schemes; see Sec. III E 2. And again the variation-after-projection scheme is the preferred method. Variation means here in general variation with respect to the single-particle wave functions in the HFB state and its occupation amplitudes $u$ and $v$. The wave functions are fixed in the model which we use later on and only the variation of $u$ and $v$ remains to be done.

\section{RPA correlations}

The correlation energy in RPA is computed with Eq. (1). The mode corresponding to particle-number phase is given by the path (23a). It is found as the zero-energy mode in the RPA spectrum because of $[\hat{H}, \hat{N}]=0$. Thus one knows already the combination $\hat{N} \equiv \hat{Q}=\left(\hat{C}^{\dagger}+\hat{C}\right) / \sqrt{2}$. The conjugate combination (3) has to be determined by the linear response $\left[\hat{H}, \hat{P}_{N}\right] \propto \hat{N}$. Once having the pair $\left(\hat{N}, \hat{P}_{N}\right)$, one can easily compute the correlation energy (1). 


\section{Simple model as test case}

\section{Model}

For further testing of the approximate scheme, we need a schematic model. It should have a gap in the single-particle spectrum to model the interplay between this gap and the pairing strength. Thus we take a two-shell model with lower band $s=-1$ and upper band $s=+1$. Each band is $N$-fold degenerated as $m=-(N-1) / 2, \ldots,+(N-1) / 2$. The states $\pm m$ are considered as the pairing conjugate partners. This yields the generalized Lipkin-Meshkov-Glick model introduced in Ref. [22]. It is simply a two-level model with seniority pairing. The model Hamiltonian reads

$$
\begin{aligned}
\hat{H}= & \epsilon \sum_{s m} s \alpha_{s m}^{\dagger} \alpha_{s m} \\
& -\chi \frac{2 \epsilon}{N}\left(\sum_{s, m>0} \alpha_{s m}^{\dagger} \alpha_{s-m}^{\dagger}\right)\left(\sum_{s, m>0} \alpha_{s-m} \alpha_{s m}\right) .
\end{aligned}
$$

We associate the following single-particle energies and occupation amplitudes:

$$
\begin{gathered}
\varepsilon_{1}=\varepsilon, \quad v_{1}=u_{-1}=u=\sqrt{1-v^{2}} \\
\varepsilon_{-1}=-\varepsilon, \quad v_{-1}=u_{1}=v .
\end{gathered}
$$

Note that the Fermi energy is $\varepsilon_{F}=0$ for symmetry reasons.

The exact solution can be obtained by diagonalizing the Hamiltonian (27) using the quasispin formalism; for details see [23].

\section{Energy in the topGOA}

The model is sufficiently simple that everything can be worked out analytically. The final result projected energy in the topGOA becomes

$$
\begin{gathered}
\frac{E_{\mathrm{BCS}}}{N \varepsilon}=-\left(\sqrt{1-(2 u v)^{2}}+\frac{\chi}{2}(2 u v)^{2}\right), \\
\delta E_{\mathrm{PNP}}=E_{\mathrm{BCS}}\left[1+(2 u v)^{2} \Lambda\left(\frac{N}{2}(2 u v)^{2}\right)\right] .
\end{gathered}
$$

This energy needs now to be compared with the BCS approximation $E_{\mathrm{BCS}}$, the RPA energy, and the exact energy.

\section{Energy in the RPA}

As shown in [23], there are two collective modes in this model. For small values of $\chi$, the mean-field approximation does not support the BCS solution and only the trivial solution with zero pairing gap $\Delta=0$ appears. In this regime, the two RPA frequencies are similar to each other; see Ref. [23] for the explicit expressions. At $\chi \sim N /(N-1)$, the system undergoes a phase transition to the superfluid phase, and the number fluctuating BCS solution becomes the ground state in the mean-field approximation. Consequently, one of the RPA frequencies becomes zero due to number conservation of the Hamiltonian (27). Applying Eq. (1) with the symmetry mode yields the RPA correlation energy

$$
\Delta E_{\mathrm{RPA}}=-\frac{\epsilon \chi}{2}
$$

The RPA frequency of the other mode is given by $2 \Delta$ $=4 \epsilon \chi(u v)$. This mode corresponds to the pairing vibration whose contribution is omitted here because we study just the projection part.

\section{Few words on the Lipkin-Nogami approach}

Full projection is often difficult, the more so if used in connection with the variation-after-projection scheme. Thus one often employs approximate schemes for particle-number projection.

A widely used approximation scheme for particle-number projection is the Lipkin-Nogami approach; see, e.g., [8] and references cited therein. It provides a good numerical approximation of the variation-after-projection scheme in situations where both HFB equations predict a collapse of the pairing correlations. The prescription of Lipkin and Nogami amounts to modifing the energy by adding the second-order Kamlah correction $\lambda_{2}(\hat{N}-\langle\hat{N}\rangle)^{2}$ where $\lambda_{2}$ is computed from mixed variances of $\hat{N}$ and $\hat{H}$; see, e.g., [20] for the SkyrmeHartree-Fock approach. The modification of the HFB equations associated with the Lipkin-Nogami prescription is obtained by a restricted variation of where $\lambda_{2}$ is not varied although its value is calculated from self-consistent expectation values. For a thorough discussion of the approximations involved see $[21,24]$. Note that the Kamlah expansion, and therefore the Lipkin-Nogami approach, uses a similar expansion as the naive GOA and does not take into account the topology of the gauge angle $\eta$.

\section{E. Results and discussion}

The upper part of Fig. 6 shows the total energy in the two-level model with seniority pairing for $N=12$ particles. Various approximations are considered. The BCS is the uncorrelated result. It decreases with constant slope up to $\chi$ $\approx 1.1$ which is the transition point from pure HF (for smaller $\chi$ ) to a truly pairing HFB state (for larger $\chi$ ). The exact energy is the goal. In addition to the RPA and topGOA, we show also the results from the Lipkin-Nogami scheme (see Sec. IV D 4). It is obvious from the figure that all corrections improve the BCS energy towards the exact result. The RPA correction works fine except for the region around the critical point. That is understandable because the critical point is distinguished by large fluctuations and the RPA is designed to be a theory for small amplitude. The Lipkin-Nogami result has a smoother trend than the RPA and corrects the energy in the wanted direction. However, the correction is incomplete, particularly at small coupling $\chi[23]$. Last but not least, the topGOA provides a very good approximation throughout all coupling strengths. It is clearly superior to the competing projection approach, the Lipkin-Nogami scheme, and it is more robust than the RPA around the transition point.

A more detailed comparison of the various approaches is shown in the lower panels of Fig. 6. It displays the correlation energies which point out the differences more clearly. 


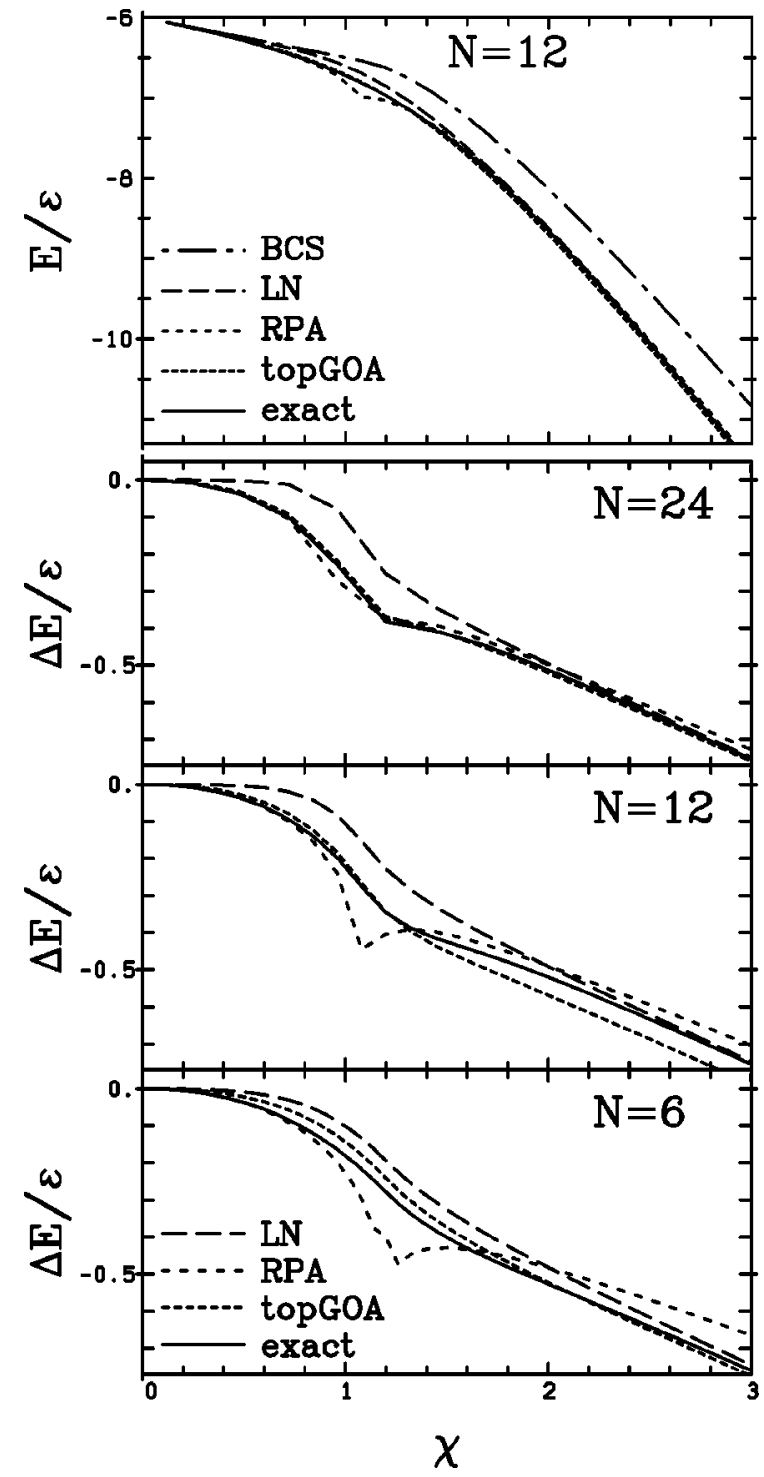

FIG. 6. Upper panel: total energies at various levels of approximation for a system with $N=12$ particles obeying the Hamiltonian (27). Lower three panels: the correlation energy $\Delta E=E-E_{\mathrm{BCS}}$ for systems with different $N$ as indicated.

First of all, the correlation energy stays about independent of system size while the total energy grows $\propto N$. This means that the relative importance of correlations shrinks as $1 / N$. This corroborates the known effect that mean-field models, here represented by BCS, become exact in the limit $N \rightarrow \infty$. The Lipkin-Nogami scheme maintains its feature to produce a "halfway" correction. It is a little bit surprising that the mismatch becomes even more pronounced with increasing system size. The RPA, on the other hand, clearly improves for larger systems. That is not surprising because mean-field theories are restored in the large- $N$ limit, and the RPA is a theory of vibrating mean fields. Finally, the topGOA provides a reliable and robust approximation to the exact correlation energy at all system sizes and coupling strengths. There are regions where it is near perfect. There are regions where one obtains visible deviations of a few percent. But the trends are always smooth and the average performance is excellent.

There are two more particularly appealing aspects of particle-number projection with the topGOA.

(1) The projected energy (26) is a closed expression in terms of expectation values of $\hat{H}$ in combination with $\hat{N}$ and of the occupation amplitudes $u$ and $v$. One can easily use that as starting point for "variation after projection." Variation with respect to the single-particle wave functions yields the appropriate correction terms to the mean-field equations. These terms can easily be incorporated in existing codes.

(2) The full GCM is not applicable in connection with nuclear density functionals, such as, e.g., the SkyrmeHartree-Fock energy. The energy density functional is given for an expectation value with one mean-field state. The extension to overlaps with different states at $q$ and $q^{\prime}$ is ambiguous. But an extension of the functional is still feasible in the immediate vicinity of a mean-field state. Thus the second-order expression $\mathcal{H}_{2}$ in Eq. (25d) can still be derived within the safe grounds of density functional theory. The topGOA thus provides a means to compute particle-number projection safely for the Skyrme-Hartree-Fock scheme.

\section{CONCLUSIONS}

We have investigated the efficient computation of ground state correlations for low-energy modes and projection. The starting point is the generator coordinate method. It is considered in the Gaussian overlap approximation which reduces the formal and numerical expense dramatically because it involves only expectation values and second-order variations therefrom. We have shown that the GOA runs into trouble in the case of weak coupling (thus broad overlaps) for coordinates with nontrivial topology. A slight modification of the scheme allows us to tune a topologically correct GOA (topGOA). We have demonstrated and tested the topGOA for two typical cases of collective coordinates: rotation-vibration and particle-number projection. To that end, we employed exactly solvable models in the spirit of the Lipkin-Meskov-Glick model.

The straightforward cases are mere projection (test cases: angular momentum and particle number). It was found that the topGOA provides an excellent approximation to full projection. Performing variation after projection (variation after projection) allows us to incorporate already a great deal of correlations into the projected states. The topGOA is particularly well suited for the variation-after-projection scheme because the projected energy is expressed in simple and compact expressions on which one can perform variation with moderate expense, far simpler than for exact projection (where nonorthogonal overlaps complicate matters). In particular for particle-number projection, the topGOA thus offers a simple and in all regimes reliable scheme which allows a thoroughly variational formulation. It is superior to the Lipkin-Nogami scheme in that respect.

Mere angular momentum projection with variation after projection was shown to grab a large portion of the correlation energy. Yet it is incomplete without the vibrational part. We have tested the topGOA for the coupled rotations and 
vibrations and it performs well in all regimes, near sphericity, at the transition point, and for well-deformed nuclei. As one could expect for a Gaussian limit, the performance improves with system size. The reverse is also true: small systems are more critical and a two-particle system is off limits.

The topGOA for vibrations involves, in principle, largeamplitude motion. This can become inconvenient in practice because a whole collective deformation path has to be mapped. The better-defined deformation of the variationafter-projection ground state allowed a small-amplitude expansion of the topGOA. The result is a scheme which can "live" with a second-order expression around the projected ground state. We consider it as a topological generalization of the random phase approximation which also deals with second-order expressions throughout and call this new scheme the topological RPA (topRPA). We find that the topRPA provides a good approximation to the results of the
topGOA and thus to the exact correlation energy for rotations and vibrations.

Altogether, we have developed with the help of topologically corrected Gaussian overlaps a palette of useful approximations for computing very efficiently the collective correlations on top of nuclear mean-field calculations. The next step is to implement that into practical calculations. Work in that direction is in progress.

\section{ACKNOWLEDGMENTS}

This work was supported in part by Bundesministerium für Bildung und Forschung (BMBF), Project No. 06 ER 808, by the U.S. Department of Energy under Grant No. DEFG06-90ER40561, and by a Grant-in-Aid for Scientific Research, Contract No. 12047203, from the Japanese Ministry of Education, Culture, Sports, Science, and Technology.
[1] P.-G. Reinhard and C. Toepffer, Int. J. Mod. Phys. E 3, 435 (1994).

[2] D.J. Rowe, Nuclear Collective Motion (Methuen, London, 1970).

[3] G.F. Bertsch and R.A. Broglia, Oscillations in Finite Quantum Systems (Cambridge University Press, Cambridge, England, 1994).

[4] P.-G. Reinhard and K. Goeke, Rep. Prog. Phys. 50, 1 (1987).

[5] P. Bonche, J. Dobaczewski, H. Flocard, P.H. Heenen, and J. Meyer, Nucl. Phys. A510, 466 (1990).

[6] K.W. Schmid and P.-G. Reinhard, Nucl. Phys. A530, 283 (1991).

[7] M. Samyn, S. Goriely, P.-H. Heenen, J.M. Pearson, and F. Tondeur, Nucl. Phys. A700, 142 (2002).

[8] H.C. Pradhan, Y. Nogami, and J. Law, Nucl. Phys. A201, 357 (1973).

[9] P.-G. Reinhard, Z. Phys. A 285, 93 (1978).

[10] A. Gozdz, K. Pomorski, M. Brack, and W. Werner, Nucl. Phys. A442, 50 (1985).

[11] H.J. Lipkin, N. Mechkov, and A.J. Glick, Nucl. Phys. 62, 188
(1965); 62, 199 (1965); 62, 211 (1965).

[12] P. Ring and P. Schuck, The Nuclear Many-Body Problem (Springer, Berlin, 1989).

[13] N. Fukuda, F. Iwamoto, and K. Sawada, Phys. Rev. 135, A932 (1964).

[14] D.J. Rowe, Phys. Rev. 175, 1283 (1968); J.C. Parikh and D.J. Rowe, ibid. 175, 1293 (1968).

[15] K. Hagino and G. Bertsch, Phys. Rev. C 61, 024307 (2000).

[16] B. Jancovici and D.H. Schiff, Nucl. Phys. 58, 678 (1964).

[17] D.M. Brink and A. Weiguny, Nucl. Phys. A120, 59 (1968).

[18] J.L. Egido and L.M. Robledo, Nucl. Phys. A494, 85 (1989).

[19] P.H. Heenen, A. Valor, M. Bender, P. Bonche, and H. Flocard, Eur. Phys. J. A 11, 393 (2001).

[20] P.-G. Reinhard, W. Nazarewicz, M. Bender, and J. A. Maruhn, Phys. Rev. C 53, 2776 (1996).

[21] H. Flocard and N. Onishi, Ann. Phys. (N.Y.) 254, 275 (1997).

[22] J. Högaasen-Feldman, Nucl. Phys. 28, 258 (1961).

[23] K. Hagino and G.F. Bertsch, Nucl. Phys. A679, 163 (2000).

[24] J. Dobaczewski and W. Nazarewicz, Phys. Rev. C 47, 2418 (1993). 